\title{
Efectos de la migración sobre el crecimiento poblacional a largo plazo de las provincias cubanas ${ }^{*}$
}

\author{
Daylin Cecilia Rodriguez Javique ${ }^{* *}$ \\ Cassio M. Turra** \\ Gabriela Marise de Oliveira Bonifácio ${ }^{* * * *}$ \\ Simone Wajnman ${ }^{* \star * *}$
}

\begin{abstract}
Cuba es un país que, además de presentar niveles de fecundidad inferiores al nivel de reemplazo, ha registrado un significativo saldo migratorio negativo. Se observa también un intenso flujo migratorio interno cuyo patrón es muy diferente entre las provincias. Debido a la magnitud de estos flujos migratorios, la migración se presenta como un componente demográfico importante en la dinámica poblacional cubana. De esta forma, el objetivo de este trabajo es estimar el efecto de la migración interna e internacional sobre el potencial de crecimiento a largo plazo de Cuba y de sus provincias. Para esto, calculamos medidas de reproducción y de crecimiento poblacional a partir de los métodos demográficos convencionales y de la metodología propuesta por Preston y Wang (2007), que se basa en los métodos de variable r. Considerando el patrón demográfico corriente, en la mayoría de los territorios de Cuba se observa una reducción substantiva en la capacidad de reposición de las generaciones y en tamaño futuro de la población cuando se consideran los efectos de la migración.
\end{abstract}

Palabras clave: Cuba. Migración internacional. Migración interna. Variable r. Población estable. Crecimiento poblacional.

\footnotetext{
* Los autores agradecen la participación de Natalia Sales Alves en el desarrollo inicial de este trabajo.

** Universidad de la Habana, Habana, Cuba (daylin@cedem.uh.cu).

${ }^{* * \star}$ Departamento de Demografía, Centro de Desenvolvimento e Planejamento Regional - Cedeplar, Universidade Federal de Minas Gerais - UFMG, Belo Horizonte-MG, Brasil (turra@cedeplar.ufmg.br).

${ }^{\star \star \star \star \star}$ Centro de Desenvolvimento e Planejamento Regional - Cedeplar, Universidade Federal de Minas Gerais - UFMG, Belo Horizonte-MG, Brasil (gabriela@cedeplar.ufmg.br).

${ }^{\star \star \star \star \star}$ Departamento de Demografía, Centro de Desenvolvimento e Planejamento Regional - Cedeplar, Universidade Federal de Minas Gerais - UFMG, Belo Horizonte-MG, Brasil (wajnman@cedeplar.ufmg.br).
} 


\section{Introducción}

Los países en vías de desarrollo han venido presentando en las últimas décadas un progresivo descenso en sus tasas de mortalidad y, más recientemente, en sus tasas de fecundidad. América Latina, que abarca una gran parte de estos países, presenta evidencias de que es escenario de uno de los más rápidos procesos de transición demográfica del mundo. Insertada en esta región, Cuba se destaca del resto de los países respecto a las especificidades que muestra en relación a sus variables demográficas. Las tasas de fecundidad y mortalidad en Cuba son extremadamente bajas, aproximándose a las tendencias observadas en los países más desarrollados. En el año 1978 la tasa de fecundidad alcanzó, por primera vez, un valor por debajo del nivel de reemplazo, comportamiento que se ha mantenido hasta la actualidad (DE ARMAS, 2008).

La persistente caída de la fecundidad crea una perspectiva de disminución del tamaño de la población a lo largo de las décadas. Junto a este hecho, se observa un movimiento migratorio internacional intenso en el país, que contribuye para que realmente la migración sea un factor determinante de la dinámica poblacional observada. Así, a partir de la década del 30 del pasado siglo, Cuba se confirma como país emisor de población, con saldos negativos que persisten, situación que la coloca dentro del contexto general, principalmente de América Latina, en un lugar importante dentro de los estudios de migración internacional (BARROS, 2002). Las características del flujo migratorio externo combinan la emigración legal definitiva y temporal, principalmente para Estados Unidos, con las salidas ilegales del territorio nacional y las visitas de emigrados cubanos de retorno temporal (CEDEM, 2009). La migración es predominantemente joven, con una tendencia a la feminización en todas las categorías. Entonces, junto con las bajas tasas de fecundidad, existe también una pérdida de mujeres en edad reproductiva que agrava la situación del crecimiento poblacional en el futuro.

Los movimientos migratorios internos también son significativos con efectos todavía poco conocidos sobre el potencial de crecimiento de cada región. En Cuba, donde los niveles de mortalidad y fecundidad en el contexto territorial son bastante homogéneos y previsibles, la migración se ha convertido en la variable demográfica capaz de explicar las desigualdades territoriales de crecimiento y la distribución espacial desigual de la población (MOREJÓN, 2007).

Algunos estudios analizan las tendencias de la migración cubana (AJA, 2002; 2007; MARTíN, 2000), pero ninguno de ellos incluye el impacto de esos movimientos migratorios en la dinámica de la población en los próximos años. Este análisis es extremadamente necesario para trazar políticas destinadas a enfrentar un posible descenso poblacional futuro que sería bien significativo, causado, principalmente, por el intenso ritmo de emigración. En este sentido, el objetivo de este trabajo es estimar el efecto de la migración internacional e interna sobre el potencial de crecimiento de largo plazo de Cuba y sus provincias, teniendo como base las condiciones demográficas en el período 2003-2007. Nuestra hipótesis es que la migración desempeña un papel importante en el crecimiento poblacional, en particular en 
un contexto de baja fecundidad y mortalidad, como es el caso de Cuba. Además, como los movimientos migratorios son diferenciales en el país, su efecto líquido sobre el crecimiento de las provincias también será diferenciado. Estos factores, en su conjunto, resultarían en medidas de crecimiento a largo plazo bastante diferentes de aquellas tradicionales obtenidas sin considerar el efecto migratorio. Así la migración interna favorecería un aumento de la población de aquellas provincias que reciben un mayor número de migrantes internos, elevando los valores de las medidas mencionadas, en detrimento de la disminución de aquellas que expulsan. Al mismo tiempo, la emigración externa sería un contrapeso al efecto de los movimientos internos, ya que las provincias que más reciben migrantes internos son las que más expulsan personas para el exterior.

Con la finalidad de introducir el efecto de la migración en el cálculo de las medidas de crecimiento a largo plazo, utilizamos la metodología propuesta por Preston y Wang (2007), que se basa en los métodos de variable r. Según esta metodología, podemos estimar la tasa neta de reproducción y la tasa intrínseca de crecimiento de una población estable equivalente, a partir de una función de tasas de crecimiento por edades y de la distribución etaria de los nacimientos por edad de la madre. Estas distribuciones reflejan tanto la fecundidad de las migrantes como de las nativas en edad reproductiva, incorporando además el efecto directo de la migración sobre el número de reproductoras. En el artículo original, los autores midieron sólo el efecto de la migración internacional en la tasa neta de reproducción de cada país. En este artículo presentamos una versión modificada, que descompone los efectos de la migración sobre la tasa neta de reproducción, considerando una separación entre la migración interna e internacional. Los datos utilizados pertenecen a los Anuarios Demográficos de Cuba entre 2002 y 2008, publicados por la Oficina Nacional de Estadística.

\section{Antecedentes}

El estudio de las tendencias demográficas a nivel mundial ha ido ganando en importancia en las últimas décadas. Las poblaciones han pasado de niveles altos de fecundidad y mortalidad a niveles bajos, lo cual ha influido directamente en la estructura por edades y ha provocado un rápido envejecimiento de la población. El caso de Cuba es peculiar; mientras mantiene niveles de fecundidad y mortalidad tan bajos (siendo un país en vías de desarrollo) como los países desarrollados, su saldo migratorio es negativo.

Esta tendencia en los saldos migratorios cubanos no es una característica nueva; desde mucho antes de la Revolución (1959) ya se observaba un comportamiento negativo en las tasas de saldo migratorio en el país. Desde la década del 1930, el número de personas que sale de Cuba excede al número de personas que entran, siendo Estados Unidos el principal receptor de migrantes cubanos. Los flujos migratorios cubanos antes del año 59 hacia este país se caracterizaron por tener múltiples naturalezas, pasando desde la migración por motivos laborales como es el caso de los tabaqueros radicados en Cayo Hueso y Tampa en el siglo XIX hasta la migración política en el siglo XX (AJA, 2009). 
Después del triunfo revolucionario, el patrón migratorio cubano cambió y se identifica un cambio en la intensidad y las características de los flujos, así como una paulatina diversificación en los destinos, a pesar de que Estados Unidos continúa siendo el principal receptor de cubanos en el exterior. En estos flujos las protagonistas suelen ser las mujeres: entre 1960 y 2006, todos los años el número de mujeres que salen de Cuba siempre ha sido mayor que el número de hombres, excepto en los primeros años de la década del 80 y en 1994, años donde las características de la emigración (por vía marítima en condiciones precarias) facilita un mayor protagonismo masculino. ${ }^{1}$ Por esta razón, obviamente, el flujo femenino hacia el exterior está afectando la fecundidad en el país, elemento que puede ser extremamente preocupante si consideramos que la fecundidad cubana muestra niveles por debajo del nivel de reemplazo desde 1978.

Entre 1960 y 2006, el saldo migratorio cubano fue negativo en lo equivalente a cerca de un millón trescientas mil personas, saldo acumulado en este período solo por vía legal y definitiva; este dato pudiera estar subestimado si tenemos en consideración que la migración ilegal, principalmente hacia Estados Unidos por vía marítima, ha sido importante en este período y que existen modalidades temporales que aumentan aún más el número de cubanos residentes en el exterior (AJA, 2009). Nótese que este patrón de baja fecundidad y emigración internacional líquida fue descrito por Preston y Wang (2007) para tan sólo algunos países de Europa Oriental. En la mayor parte del mundo desarrollado los flujos migratorios actúan en sentido contrario, lo cual minimiza el efecto de la baja fecundidad en el crecimiento a largo plazo.

Analizando lo que sucede en el interior del país, los flujos migratorios tanto nacionales como internacionales son diferentes entre las provincias. Mientras las provincias orientales son emisoras de población hacia las provincias occidentales y desde el punto de vista de migración interna son las mayores emisoras de población para dentro del país, éstas son las que menos emiten población para fuera del país; no obstante, también han perdido población en los últimos años (teniendo en consideración la provincia de residencia del migrante internacional en el momento de su salida del país). Las provincias occidentales (excepto Pinar del Río), por su parte, ganan población constantemente por concepto de migración interna pero son las que más pierden hacia fuera del país (MONTES, 2000). Como se muestra en la Tabla 1, por ejemplo, en la Ciudad de La Habana la tasa neta de migración interna era de 5,4 por mil, sin embargo la tasa neta de migración total era de -3.1 por mil.

Con estos datos es obvio el impacto de la migración internacional en esta provincia, que, incluso con una tasa neta de migración interna relativamente alta, todavía está perdiendo personas por concepto de migración. De la misma manera, en el resto de las provincias, aunque en menor medida, la migración internacional presenta comportamientos similares,

\footnotetext{
${ }^{1}$ En el año 1980, Cuba liberó un puerto situado en la ciudad de Mariel, cerca de la ciudad de La Habana para que todas las personas que quisieran abandonar el país por vía marítima lo hicieran, una situación similar pero en menor dimensión ocurrió en la década de los 90 s.
} 
incluso en las provincias en que la tasa neta de migración total aún es positiva, pero siempre presenta valores inferiores a los de la tasa neta de migración interna. Considerando que en todos los territorios del país la tasa global de fecundidad está por debajo del nivel de reemplazo, se hace necesario saber de qué manera la fecundidad y la migración afectan en su conjunto el crecimiento a largo plazo del país y sus provincias.

TABLA 1

Tasa global de fecundidad, tasa neta de migración interna y tasa neta de migración total Cuba y provincias - 2008

\begin{tabular}{lccc}
\hline \multicolumn{1}{c}{ Provincias } & $\begin{array}{c}\text { Tasa global de } \\
\text { fecundidad }\end{array}$ & $\begin{array}{c}\text { Tasa neta de migración } \\
\text { interna }\end{array}$ & $\begin{array}{c}\text { Tasa neta de migración } \\
\text { total }\end{array}$ \\
\hline Pinar del Río & 1,6 & $-2,0$ & $-5,1$ \\
La Habana & 1,5 & 6,2 & 3,1 \\
Ciudad de La Habana & 1,3 & 5,4 & $-3,1$ \\
Matanzas & 1,3 & 5,3 & 2,8 \\
Villa Clara & 1,5 & $-1,7$ & $-4,8$ \\
Cienfuegos & 1,6 & 3,1 & 0,6 \\
Sancti Spíritus & 1,7 & 0,5 & $-1,4$ \\
Ciego de Ávila & 1,6 & 1,5 & $-0,6$ \\
Camagüey & 1,5 & $-2,8$ & $-5,6$ \\
Las Tunas & 1,6 & $-1,8$ & $-3,0$ \\
Holguín & 1,8 & $-3,5$ & $-4,6$ \\
Granma & 1,7 & $-3,8$ & $-4,4$ \\
Santiago de Cuba & 1,7 & $-4,7$ & $-6,2$ \\
Guantánamo & 1,9 & $-7,0$ & $-8,2$ \\
Isla de la Juventud & 1,7 & $-8,3$ & $-11,0$ \\
Cuba & 1,6 & - & $-3,3$ \\
\hline
\end{tabular}

Fuente: Anuario Demográfico de Cuba, 2008.

\section{Metodología}

Con el objetivo de evaluar el impacto directo de la migración sobre el crecimiento a largo plazo de las provincias cubanas, calculamos la tasa neta de reproducción (TNR) según cuatro escenarios distintos: i) sin efecto de migración (TNR); ii) considerando solamente el efecto líquido de la migración interna (TNRMN); iii) considerando el efecto de la migración líquida total (TNR ${ }^{M T}$ ) $y$ finalmente iv) considerando apenas el efecto de la migración internacional (TNR ${ }^{M I}$ ). Además, estimamos el tiempo en años para que la población se reduzca a la mitad después de haber sido alcanzada la estructura etaria equivalente, contrastando los dos escenarios: con ausencia de los efectos directos de la migración y el papel de la migración total.

Convencionalmente, la tasa neta de reproducción (TNR) equivale al número medio de hijas nacidas vivas de una mujer, representativa de una cohorte hipotética, que sobrevive hasta el final de su período reproductivo y experimenta las tasas específicas de mortalidad y maternidad vigentes en un determinado período. La TNR convencional es una medida de largo plazo que se refiere al reemplazo entre generaciones y que ignora los efectos de las migraciones sobre el tamaño de la cohorte hipotética: 
$T N R=\int_{0}^{\infty} p(a) m(a) d a$

Donde $p(a)$ es la probabilidad de supervivencia desde el nacimiento hasta la edad $a, y$ $m(a)$ es la función de maternidad.

A pesar de que la TNR convencional no incorpora efectos directos de la migración, representados por el aumento o disminución de la población en edad reproductiva, ella refleja los efectos indirectos de la migración, representados por las variaciones en la función de fecundidad en la población analizada. Estimamos la TNR convencional a partir de las funciones de mortalidad y maternidad vigentes en Cuba. En escala directa, estimamos la TNR en cada provincia (PRESTON et al., 2000) de la siguiente forma:

$T N R=\frac{1}{l_{0}} \sum_{x=\alpha}^{\beta-n}{ }_{n} F_{x}^{F} \cdot{ }_{n} L_{x}^{F}$

Donde $l_{0}$ es el número de nacidas vivas en la tabla de vida, $n$ es la tasa específica de maternidad; $y_{n} L_{x}^{F}$ representa el número de personas-año entre las edades $x$ y $x+n$ de una cohorte hipotética que experimentó la función de mortalidad dada por la tabla de vida femenina del período.

Calculamos las tasas específicas de maternidad para cada grupo etario dentro del ciclo de reproducción en base a las informaciones del total de nacidas vivas en cada grupo de edad entre 2003-2007 para el numerador y la población femenina estimada para el medio del período en el denominador. Los datos de nacimientos se obtuvieron a través de las “Inscripciones de Nacimiento" emitidas por el Ministerio de Salud Pública y el Registro del Estado Civil del Ministerio de Justicia. Estos datos se caracterizan por tener una excelente cobertura fundamentalmente por dos razones: por un lado, a partir de octubre de 1967 se estableció como ley la obligatoriedad del registro del recién nacido en los 20 días siguientes al parto; por otro, casi la totalidad de los nacimientos ocurren en centros del sistema de salud. La población es estimada al cierre de cada año a partir de la ecuación compensadora tomando como base la población registrada en el Censo de Población y Vivienda realizado en Cuba en septiembre del 2002 y utilizando las estadísticas vitales (muertes y nacimientos) y migraciones. Obviamente, la población es estimada por sexo y edad en cada provincia, municipio y zona del país.

Además, fueron construidas tablas de vida para obtener las funciones de supervivencia por grupo de edad. Para esto se utilizó la información de defunciones femeninas en el período estudiado. Las defunciones son captadas a partir del "Certificado Médico de Defunción” y el "Certificado de Defunción Neonatal”, emitidos por el Ministerio de Salud Pública y los Registros del Estado Civil. A partir de 1986, con la Ley 51, se establecen las funciones y atribuciones de los Registros del Estado Civil como entidad autorizada para la inscripción de las defunciones. Con este hecho se plantea un reordenamiento y organización en el registro de las defunciones y por tanto ello contribuye significativamente a la ampliación, calidad y cobertura de estas estadísticas. ${ }^{2}$

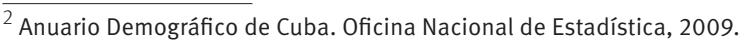


Como uno de los objetivos de este artículo es evaluar los efectos aislados de cada uno de los flujos migratorios sobre el potencial de crecimiento a largo plazo de la población, en el segundo conjunto de estimaciones se intentó medir tasas netas de reproducción que reflejaran también la migración. Preston y Wang (2007) demostraron que el concepto convencional de la TNR puede ser extendido a través de la inclusión de la función de crecimiento o decrecimiento de la cohorte hipotética desde el nacimiento hasta la edad $a$ como consecuencia de las migraciones:

$$
T N R^{M T}=\int_{0}^{\infty} p(a) p^{\star}(a) m(a) d a
$$

Donde $p(a)$ es la probabilidad de supervivencia desde el nacimiento hasta la edad $a, p^{*}(a)$ es la función de crecimiento o decrecimiento de la cohorte hipotética desde el nacimiento hasta la edad $a$ como consecuencia de las migraciones y $m(a)$ es la función de maternidad.

Teniendo como base la ecuación (3), introdujimos inicialmente un término específico para las migraciones internas:

$$
T N R^{M N}=\int_{0}^{\infty} p(a) p^{M N}(a) m(a) d a
$$

Donde $p^{\mathrm{MN}}(a)$ es la función de crecimiento o decrecimiento de la cohorte hipotética del nacimiento hasta la edad a debido a las migraciones internas.

Para esto utilizamos datos de los movimientos migratorios internos, captados a través de estadísticas continuas establecidas a partir de 1960 con la implantación del Registro Nacional de Consumidores perteneciente al Ministerio de Comercio Interior. Las informaciones sobre migraciones internas son publicadas en tres grandes grupos (0-14; 15-59; 60 y más), pero fue necesario además distribuir los datos entre grupos etarios quinquenales. El número absoluto y relativo de migrantes por edades depende del riesgo de migrar en cada edad y de la distribución etaria de la población expuesta al riesgo. Como no conocemos el riesgo en cada edad, la distribución de los migrantes dentro de cada gran grupo de edad fue realizada según el peso de cada grupo quinquenal en la población femenina en medio del período. Es válido aclarar que desde el punto de vista de la variable de interés en el trabajo (crecimiento demográfico), el mayor problema sería no conocer el número de migrantes que están fuera del período reproductivo. Felizmente, este no fue un inconveniente en nuestro trabajo, ya que sabemos cuántos, del total de migrantes, estaban por encima de los 59 años (edad muy próxima al límite superior del período reproductivo).

Disponiendo de información detallada sobre flujos migratorios internos por grupos de edad, sexo y provincia fue posible construir nuevas tablas de vida, combinando la información del número de defunciones en cada grupo y el saldo migratorio interno correspondiente (inmigrantes menos emigrantes) en una función de supervivencia equivalente a la combinación de las funciones $\mathrm{p}(\mathrm{a})$ e $\mathrm{p}^{\mathrm{MN}}$ (a), descritas en (4). Para estimar esta nueva función de supervivencia femenina con los efectos de la migración interna, fueron calculadas inicialmente tasas específicas por edad para las mujeres, en las cuales el numerador es igual a 
la diferencia entre las muertes y el saldo migratorio (diferencia entre el número de inmigrantes y el número de emigrantes) y el denominador es igual a la población en el medio del período:

$$
{ }_{n} M_{x}^{F}=\frac{{ }_{n} D_{x}^{F}-\left({ }_{n} I_{x}^{F}-{ }_{n} E_{x}^{F}\right)}{{ }_{n} N_{x}^{F}}
$$

Donde ${ }_{n} M_{x}^{F}$ es la tasa específica por edades, ${ }_{n} D_{x}^{F}$ es el número de defunciones femeninas por edad, ${ }_{n} I_{x}^{F}$ e ${ }_{n} E_{x}^{F}$ es el número de inmigrantes y emigrantes mujeres por edad, y ${ }_{n} N_{x}^{F}$ es la población femenina de cada grupo de edad del medio del período.

A través de la ecuación 6, las tasas de período fueron convertidas a probabilidades de cohorte, asumiendo que las personas mueren y migran, en promedio, en la mitad del intervalo de edad (PRESTON et al. 2000).

$$
{ }_{n} p_{x}=1-{ }_{n} q_{x}=1-\left\lfloor\frac{n \cdot{ }_{n} M_{x}^{F}}{1+\frac{n}{2} \cdot{ }_{n} M_{x}^{F}}\right\rfloor
$$

En base a la nueva función de supervivencia por edades, fue estimada la nueva función del número de personas-año por edades $\left({ }_{n} L_{x}^{F}\right)$, que considera la mortalidad y la migración interna simultáneamente. A través de esa información de supervivencia y de las tasas de maternidad específicas por edades, se obtuvo el valor de la TNR ${ }^{\mathrm{MN}}$.

El tercer conjunto de estimativas considera el efecto de la migración líquida total. En este caso, ya que nosotros no teníamos informaciones directas precisas sobre la migración internacional que podrían combinarse con los datos de la migración interna, no se podía usar la estrategia anterior para calcular la TNR a partir de las funciones de maternidad y de la tabla de vida ajustadas con las migraciones. Así, la alternativa que encontramos fue aplicar la metodología desarrollada por Preston y Wang (2007), basada en el método de la variable-r, que utiliza las tasas específicas de crecimiento por edad y la distribución de los hijos según grupo de edad de la madre al tener esos hijos para estimar la TNR y la tasa intrínseca de crecimiento. Como este método parte de distribuciones observadas, que incorporan toda la historia demográfica de cada cohorte, captan también los efectos netos directos de la migración total sobre el tamaño de la población en edad reproductiva. Por tanto, siguiendo a Preston y Wang (2007), la TNR ${ }^{\mathrm{MT}}$ puede ser calculada como:

$T N R^{M T}=\int_{0}^{\infty} v(a) e^{\int_{0}^{a} r(x) d x} d a$

En la cual $v_{(a)}$ es la proporción de mujeres según el grupo de edad en que ellas tuvieron sus hijos entre 2003 y 2007; $\mathrm{r}_{(\mathrm{x})}$ representa la tasa de crecimiento de la población femenina para cada grupo quinquenal de mujeres en el período en cuestión.

Asumiendo que las tasas de reproducción neta con y sin migración son aditivas, se puede estimar la TNR ${ }^{\mathrm{MI}}$, abarcando el efecto de la migración internacional, a partir de las tasas de reproducción netas calculadas anteriormente, según la fórmula (8). 
$T N R^{M I}=T N R-\left[\left(T N R-T N R^{M T}\right)-\left(T N R-T N R^{M N}\right)\right]$

Después de estimar estos valores, cada provincia exhibía un conjunto de cuatro valores de TNR, representativos de situaciones diferentes que consideraban la migración como elemento importante de la dinámica demográfica para el período 2003-2007. Para mostrar mejor el efecto directo de la migración total sobre el crecimiento a largo plazo de la población cubana, estimamos el tiempo en años para que la población se reduzca a la mitad después de haber sido alcanzada la estructura etaria equivalente a las funciones demográficas observadas, según dos escenarios: sin migración y con el efecto de la migración total. Para eso, calculamos primero las tasas intrínsecas de crecimiento asumiendo un intervalo intergeneracional de 30 años. ${ }^{3}$ Seguimos la estrategia sugerida por Coale (1957) y resolvimos la siguiente ecuación para cada uno de los escenarios:

$r_{0}=\frac{\operatorname{Ln}(T N R)}{30}$

Por iteración, repetimos el cálculo de $r_{0}$ hasta que la ecuación de Lotka 4 se aproximase lo máximo posible al valor de 1.

Asumiendo una tasa de crecimiento compuesta continuamente en cada uno de los escenarios, empleamos la expresión genérica presentada por Keyfitz y Caswell (2005), para calcular el tiempo en años para que la población estable equivalente se reduzca a la mitad $T=\frac{\operatorname{Ln}(0,5)}{r}$

Es importante aclarar que nuestras estimaciones describen lo que pudiera suceder tanto con el reemplazo generacional como con el crecimiento de la población en Cuba en un contexto de estabilidad. Es decir, las medidas calculadas serían notables sólo en un medio y largo plazo, con la fijación de las actuales funciones de fecundidad, mortalidad y migraciones. En este sentido, esas medidas son apenas indicadores de posibles escenarios futuros.

\section{Resultados}

La Tabla 2 presenta los valores de la tasa neta de reproducción (TNR) calculadas en los cuatro escenarios propuestos en este estudio para cada provincia cubana, ordenados de forma descendiente en función del impacto de la migración total en la tasa neta de reproducción. En primer lugar se observa que, en todas las provincias, la TNR convencional es menor que 1; esto significa que, en todo el país, la generación de hijas es relativamente menor que la

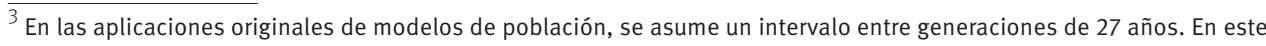
artículo adoptamos un período un poco más largo, de 30 años, de forma que nuestros resultados se tornen consistentes con los de otros países del mundo, estimados por Preston y Wang (2007).

${ }^{4}$ En el escenario sin migración, usamos $1=\int_{\alpha}^{\beta} e^{-r a} p(a) m(a) d a$. En el escenario con el efecto de la migración total, usamos Preston y Wang (2007), $1=\int_{0}^{\infty} e^{-r a} v(a) e^{\int_{0}^{a} r(x) d x} d a$
} 
generación de madres, o sea, no hay una reposición de una generación por la otra cuando se considera apenas el efecto de la fecundidad y de la mortalidad.

TABLA 2

Tasas netas de reproducción con y sin la contribución de la migración Cuba y provincias - 2003-2007

\begin{tabular}{|c|c|c|c|c|c|}
\hline \multirow[b]{2}{*}{ Provincias } & \multicolumn{4}{|c|}{ TNR } & \multirow{2}{*}{$\begin{array}{c}\text { Cambio } \\
\text { en la TNR } \\
\text { convencional } \\
\text { considerando } \\
\text { la migración } \\
\text { total }\end{array}$} \\
\hline & Convencional & $\begin{array}{l}\text { Considerando } \\
\text { migración } \\
\text { interna }\end{array}$ & $\begin{array}{c}\text { Considerando } \\
\text { migración } \\
\text { internacional }\end{array}$ & $\begin{array}{l}\text { Considerando } \\
\text { migración total }\end{array}$ & \\
\hline Guantánamo & 0,83 & 0,72 & 0,78 & 0,67 & $-0,17$ \\
\hline Isla de la Juventud & 0,79 & 0,69 & 0,71 & 0,62 & $-0,17$ \\
\hline Pinar del Río & 0,77 & 0,75 & 0,69 & 0,66 & $-0,11$ \\
\hline Granma & 0,80 & 0,74 & 0,76 & 0,70 & $-0,10$ \\
\hline Ciudad de La Habana & 0,63 & 0,68 & 0,49 & 0,54 & $-0,09$ \\
\hline Holguin & 0,80 & 0,76 & 0,76 & 0,71 & $-0,09$ \\
\hline Santiago de Cuba & 0,74 & 0,67 & 0,72 & 0,65 & $-0,09$ \\
\hline Camagüey & 0,68 & 0,66 & 0,63 & 0,61 & $-0,08$ \\
\hline Villa Clara & 0,69 & 0,67 & 0,62 & 0,61 & $-0,08$ \\
\hline Las Tunas & 0,70 & 0,67 & 0,68 & 0,65 & $-0,05$ \\
\hline Cienfuegos & 0,75 & 0,77 & 0,69 & 0,72 & $-0,02$ \\
\hline Sancti Spíritus & 0,68 & 0,69 & 0,65 & 0,67 & $-0,01$ \\
\hline Ciego de Avila & 0,70 & 0,74 & 0,67 & 0,71 & 0,01 \\
\hline Matanzas & 0,67 & 0,75 & 0,64 & 0,73 & 0,06 \\
\hline La Habana & 0,70 & 0,85 & 0,65 & 0,80 & 0,10 \\
\hline Cuba & 0,72 & & 0,65 & 0,65 & $-0,06$ \\
\hline
\end{tabular}

Fuente: Anuarios Demográficos de Cuba, 2003-2007.

Además de esto, al introducir el efecto directo de la migración sobre el número de mujeres hasta el final del período reproductivo, el impacto en los valores de la TNR es diferenciado por provincias. Las provincias La Habana, Matanzas y Ciudad de La Habana (capital del país), que están situadas en la parte occidental de la isla, se caracterizan por tener saldos migratorios internos positivos (Tabla 1). Una situación muy similar, pero en menor medida, la observamos en las tres provincias de la región central, Cienfuegos, Sancti Spiritus y Ciego de Ávila. En estas provincias, manteniendo el patrón actual de la migración interna, las TNRs se tornarían ligeramente mayores que la medida convencional calculada considerando tan sólo la fecundidad y la mortalidad; no obstante, se encontraría por debajo del nivel de reemplazo. Por otro lado la región oriental del país, específicamente las provincias de Holguín, Granma, Santiago de Cuba y Guantánamo, incluyendo el municipio especial Isla de la Juventud, se caracterizan por ser emisores de población hacia otras provincias del país (MOREJÓN, 2007). Este comportamiento se observa también en las provincias Pinar del Río, Villa Clara, Camagüey y Las Tunas. De esta forma, en todas estas provincias la migración interna genera una reducción en el valor de la TNR, en función de la pérdida de mujeres en edad reproductiva en estos territorios, acentuando los efectos de la baja fecundidad sobre el ritmo de reposición 
de las generaciones. La variación en los valores de la TNR considerando el efecto directo de la migración interna sugiere que a largo plazo, manteniéndose este patrón, los flujos migratorios tendrían un impacto sobre la distribución espacial de la población cubana, a través de su efecto sobre el reemplazo de las generaciones, mayor de lo que sería esperado.

La dinámica poblacional en Cuba presenta una característica que la distingue de otras poblaciones. Existe una relación entre los procesos de migración interna y externa que complementa los movimientos migratorios (AJA, 2007). Los principales flujos de migración externa son producidos en la zona occidental del país, que a su vez es receptora de la migración interna del país. Coherente con esta tendencia, los resultados muestran que en las principales provincias de destino de migrantes internos, el impacto de la migración internacional reduce la TNR.

Por una parte ganan población femenina en edad reproductiva por concepto de migración interna, sin embargo el flujo hacia el exterior hace que la fecundidad sea aún más baja. Por ejemplo, en Ciudad de La Habana, principal centro emisor de migrantes internacionales, se observa el mayor efecto de los flujos internacionales con una reducción de 0,14 hijas por mujer en su TNR convencional, elemento que vislumbra una pérdida significativa de mujeres en edad reproductiva para el exterior del país procedentes de esta provincia. En contraposición, si observamos el efecto de la migración interna, en esta misma provincia la TNR convencional aumenta 0,05 hijas por mujer. En otras provincias, como Pinar del Río, Villa Clara, Camagüey y Las Tunas, la migración interna e internacional actúa en el mismo sentido, contribuyendo a la reducción de la TNR convencional también de forma substantiva.

La última columna de la Tabla 2 muestra el efecto total de la migración sobre las tasas netas de reproducción. El efecto directo de la migración total disminuye el valor de la TNR convencional en la mayoría de las provincias del país, indicando que el flujo migratorio apunta, a largo plazo, a una mayor reducción del nivel de reemplazo de las generaciones. Este patrón también es válido para la población total de Cuba. Vale la pena llamar la atención sobre el hecho de que las provincias La Habana, Matanzas y Ciego de Ávila representan una excepción en este sentido, donde el flujo de inmigrantes internos compensa la pérdida de mujeres hacia el exterior, haciendo que la TNR convencional aumente. Sin embargo, es necesario resaltar que, incluso en estas provincias en que la migración tiene un impacto positivo sobre el crecimiento a largo plazo, las TNR todavía permanecen por debajo del nivel de reemplazo.

Los diferentes impactos de la migración total sobre el crecimiento poblacional a largo plazo en Cuba también se reflejan en los valores de las tasas intrínsecas de crecimiento, obtenidas para cada provincia. La tasa convencional expresa tan sólo el crecimiento intrínseco a las funciones de maternidad y mortalidad. Por otro lado, la tasa intrínseca de crecimiento en presencia de migración es una medida de la velocidad que una población experimenta en su crecimiento, si las tasas específicas de fecundidad, mortalidad y migración por edades se mantuvieran a largo plazo. En un ejercicio de previsión hipotética, estas medidas son presentadas en este trabajo en función de la cantidad de años que tarda la población en reducirse a la mitad, después de haber alcanzado la estructura etaria estable implícita 
correspondiente a las funciones demográficas observadas (Tabla 3), con y sin efecto de la migración.

Como puede ser observado en la Tabla 3, cuando no se considera el efecto de la migración total la mayoría de las provincias tendría su población reducida a la mitad en menos de una generación ${ }^{5}$. Un tiempo relativamente corto, como consecuencia de los bajos niveles de fecundidad del país. Considerando el efecto de la migración total, esta reducción sucedería de forma todavía más rápida para la mayor parte de las provincias. Guantánamo es uno de los ejemplos más evidentes, considerando el efecto de la migración; se reduciría el tiempo en un 55\% para que la población decreciera a la mitad pasando de 100 a 45 años. La provincia Ciudad de La Habana muestra el tiempo más corto de reducción, tardaría solamente 30 años para reducir a la mitad su población considerando el efecto migratorio. Tal escenario se reflejaría en el país como un todo; se llevaría tan sólo 43 años para que la población se redujera a la mitad, una vez alcanzada la estabilidad a partir de las condiciones demográficas actuales.

Todos estos resultados sugieren que la migración juega un papel significativo en la dinámica demográfica de las provincias cubanas y por tanto para el total del país. Este efecto debe ser analizado con cuidado pues el resultado contribuye a potenciar la disminución de la población cubana en general y en muchas de las provincias en un período de tiempo menor que el que normalmente llevaría sin el impacto de la migración.

TABLA 3

Tiempo en años para que la población se reduzca a la mitad, después de haber sido alcanzada la estructura etaria equivalente a las funciones demográficas observadas Cuba y provincias

\begin{tabular}{lcc}
\hline \multicolumn{1}{c}{ Provincias } & Sin migración & $\begin{array}{c}\text { Con efecto de la } \\
\text { migración total }\end{array}$ \\
\hline Guantánamo & 100 & 45 \\
Isla de la Juventud & 77 & 38 \\
Pinar del Río & 72 & 45 \\
Granma & 83 & 50 \\
Ciudad de La Habana & 41 & 30 \\
Holguín & 82 & 53 \\
Santiago de Cuba & 62 & 43 \\
Camagüey & 48 & 36 \\
Villa Clara & 49 & 37 \\
Las Tunas & 51 & 41 \\
Cienfuegos & 63 & 56 \\
Sancti Spíritus & 48 & 46 \\
Ciego de Ávila & 50 & 53 \\
Matanzas & 45 & 58 \\
La Habana & 50 & 82 \\
Cuba & 55 & 43 \\
\hline
\end{tabular}

Fuente: Anuarios Demográficos de Cuba, 2003-2007.

\footnotetext{
$\overline{5}$ Considerando que una generación dura, en media, 80 años.
} 


\section{Consideraciones finales}

Cuba es un país latinoamericano que se destaca del resto de los países de la región por las especificidades demográficas que presenta. Uno de los principales factores demográficos que ha llamado la atención es la baja fecundidad de las cubanas y la homogeneidad entre las diferentes regiones dentro del país en este aspecto. Unido a esta realidad, el país muestra movimientos migratorios que, como ha sido demostrado por los datos expuestos en el trabajo, influyen fuertemente en las medidas de crecimiento de largo plazo de la población. Los resultados encontrados en este trabajo indican que, si se mantienen los patrones demográficos actuales, en cada generación nacerá un número de hijas insuficiente para reponer la generación de madres, los cual tendría implicaciones importantes en cuanto a número de habitantes y estructura etaria.

La migración, por su parte, se inserta en ese contexto como un factor agravante de la situación. Mujeres en edad reproductiva salen de sus provincias de origen en dirección a otras provincias, hecho que acaba favoreciendo las provincias de destino en el escenario de la migración interna. Con esto, cuando se observa tan sólo el efecto de la migración interna, algunas provincias cubanas tienen una velocidad de disminución de su población menor que otras. Sin embargo, estas mismas provincias que parecían ser favorecidas por la atracción de migrantes internos son aquellas que, en su mayoría, expulsan una mayor cantidad de mujeres en edad reproductiva para otros países. Así, a pesar de recibir migrantes internos, la intensidad de la migración internacional torna su situación demográfica un tanto más compleja.

De este modo, los resultados presentados en este trabajo, además de enfatizar la relevancia de la inclusión de la migración en los estudios de las demás variables demográficas como factor interviniente en el crecimiento de la población, plantean una cuestión que debe ser considerada actualmente en el país: ¿Qué hacer para que la población cubana no disminuya significativamente de tamaño en el futuro? El debate acerca de esta pregunta afecta al país no sólo en la esfera demográfica, sino también en otras esferas que influyen en la población, como por ejemplo el sector económico. Bajas tasas de fecundidad, junto con saldos migratorios negativos, principalmente en edades jóvenes, trae consigo una pérdida de mano de obra que es indispensable para la economía, ya que el número de personas en estas edades es cada vez menor. Otro elemento importante se refiere a que, dada la fase avanzada de la transición demográfica en la que Cuba se encuentra, el contingente de adultos mayores está aumentando cada vez más. Esto unido a la reducción del número de personas aptas para sustentar este grupo al final de la vida puede resultar en un hecho preocupante que puede generar serios problemas para la economía cubana.

Se hace necesario elaborar políticas públicas enfocadas en la fecundidad y la migración internacional en el país, a fin de proporcionar un comportamiento reproductivo más favorable para el crecimiento poblacional junto con un incentivo de la permanencia de esas mujeres dentro del territorio nacional. No podemos dejar de mencionar que políticas que incentiven 
una inmigración ya sea de retorno o de personas no nativas, puede ayudar a reducir la velocidad de la reducción del tamaño poblacional en Cuba.

El presente estudio suscita también una serie de cuestionamientos que pueden auxiliar en el tratamiento de la situación actual del país. En primer lugar sería oportuno reflexionar acerca de los factores que están determinando la migración interna y externa en Cuba, teniendo en cuenta las diferencias territoriales. Por otro lado pudiera ser interesante indagar acerca de la fecundidad de las mujeres cubanas migrantes y compararla con la fecundidad de las no migrantes. Reflexionar acerca de cuestiones como éstas podría contribuir a un mejor entendimiento de la situación y del comportamiento de estos dos componentes demográficos en el país y ayudaría a una mejor implementación de políticas sociales eficientes.

Finalmente, con este trabajo no pretendemos desarrollar ninguna crítica a los estudios realizados antes, ni tampoco interferir en alguna teoría sobre el asunto. Lo que realmente buscamos es enfatizar el papel que la migración está ejerciendo en el análisis de medidas de crecimiento a largo plazo como lo son la Tasa Neta de Reproducción y la Tasa Intrínseca de Crecimiento a través de la aplicación de una metodología ya elaborada. Los resultados obtenidos revelan una realidad que, aunque ya parecía casi obvia, era hasta entonces poco conocida o poco comprobada. Sería interesante aplicar esta misma metodología para otros países de América Latina para indicar si lo que ocurre en Cuba forma parte de una tendencia globalizada para países en vías de desarrollo o es otra especificidad de la realidad cubana. Es posible que en países como Brasil, por ejemplo, la reducción generalizada de la fecundidad en ciudades pequeñas del interior, que también son más susceptibles a perder población por migración en edad reproductiva, genere vacíos poblacionales en un futuro, con notables consecuencias para la dinámica económica y social (WAJNMAN; TURRA; DUARTE, 2013). En este sentido, el tipo de análisis aquí empleado puede ser una herramienta de planificación a largo plazo, al indicar las áreas que están expuestas a un mayor riesgo futuro de decrecimiento poblacional.

\section{Referencias}

AJA, A. Al cruzar las fronteras. La Habana: Centro de Estudios Demográficos, Universidad de La Habana, 2009.

Tendencias y retos de Cuba ante el tema de la emigración. Fondos del Centro de Estudios de Migraciones Internacionales. La Habana: Centro de Estudios de Migración Internacional, Universidad de La Habana, 2002.

La migración desde Cuba. Aldea Mundo, v. 11, n. 022, 2007.

OFICINA NACIONAL DE ESTADÍSTICA. Anuarios Demográficos de Cuba. Cuba: ONE, 2003-2009.

BARROS, O. Escenarios demográficos de la población cubana. 2000-2050. La Habana: Ciencias Sociales, 2006.

CEDEM - Centro de Estudios Demográficos. Cuba: población y desarrollo. La Habana: CEDEM, Universidad de La Habana, 2009.

COALE, A. A new method for calculating Lotka's r-The intrinsic rate of growth in a stable population.

Population Studies, v.11, n. 1, p. 92-4, 1957. 
DE ARMAS, M. No es lo mismo pero es igual: a singularidade da segunda transição demográfica em Cuba. Tesis en opción del grado de Doctor en Demografía. Centro de Desenvolvimento e Planejamento Regional, Universidade Federal de Minas Gerais, Brasil, 2008.

KEYFITZ, N.; CASWELL, H. Applied mathematical demography. New York: Springer, 2005.

MARTín, C. Cuba: vida cotidiana, familia y emigración. Tesis en opción de grado de Doctor en Ciencias Psicológicas, Centro de Migraciones Internacionales, Universidad de La Habana, Cuba, 2000.

MONTES, N. Una aproximación al estudio de la migración interna en Cuba. Temas, v. 20-21, p. 135146, 2000.

MOREJÓN, B. Características diferenciales de los migrantes internos en Cuba. Novedades de Población, v. 3, n. 6, p, 26-44, 2007.

PRESTON, S.; WANG, H. Intrisic growth rates and net reproduction rates in the presence of migration. Population and Development Review, v. 33, n. 4, p. 357-366, 2007.

PRESTON, S.; HEUVELINE, P.; GUILLOT, M. Demography - Measuring and modeling population processes. Massachussets: Blackwell Publishers, 2000.

WAJNMAN, S; TURRA, C. M.; PINHO, B. A. T. D. A migração nos modelos de populações teóricas. Belo Horizonte: Centro de Desenvolvimento e Planejamento Regional - Cedepar, Universidade Federal de Minas Gerais - UFMG, 2013. Mimeografado.

\section{Autores}

Daylin Cecilia Rodriguez Javique es maestra en Demografía por el Centro de Desenvolvimento e Planejamento Regional - Cedepar, Universidade Federal de Minas Gerais - UFMG. Profesora del Centro de Estudios Demográficos de la Universidad de La Habana.

Cassio M. Turra es doctor en Demografía por la Universidad de la Pennsylvania. Profesor adjunto del Departamento de Demografía de la Universidade Federal de Minas Gerais - UFMG.

Gabriela Marise de Oliveira Bonifácio es maestra en Demografía por el Centro de Desenvolvimento e Planejamento Regional - Cedepar, Universidade Federal de Minas Gerais - UFMG. Estudiante de Doctorado en Demografía - Cedeplar/UFMG.

Simone Wajnman es doctora en Demografía por el Centro de Desenvolvimento e Planejamento Regional - Cedepar, Universidade Federal de Minas Gerais - UFMG. Profesora asociada del Departamento de Demografía de la UFMG.

\section{Resumo}

Efeitos da migração sobre o crescimento populacional de longo prazo das províncias cubanas

Cuba é um país que, além de apresentar níveis de fecundidade inferiores ao nível de reposição, tem registrado um saldo migratório líquido negativo significativo. Observa-se também um intenso fluxo migratório interno, cujo padrão é bastante diferente nas diversas províncias do país. Devido à magnitude destes fluxos migratórios, a migração apresenta-se como um componente demográfico importante na dinâmica populacional cubana. Assim, o objetivo deste trabalho é estimar o efeito das migrações interna e internacional sobre o potencial de crescimento de longo prazo de Cuba e de suas províncias. Para tanto, foram calculadas medidas de reprodução e de crescimento populacional a partir dos métodos demográficos convencionais e da metodologia proposta por Preston e Wang (2007), que se baseia nos métodos da variável-r. Levando em conta o padrão demográfico atual, observa-se, na maioria dos 
territórios de Cuba, uma redução substantiva na capacidade de reposição das gerações e no tamanho futuro da população quando se consideram os efeitos da migração.

Palavras-chave: Cuba. Migração internacional. Migração interna. Variável-r. População estável. Crescimento populacional.

\section{Abstract}

\section{Effects of migration on long-term population growth in the provinces of Cuba}

Cuba has shown very low fertility levels, even below replacement level, in addition to significant negative net migration flows. In the country's interior there is intense population mobility, characterized by different migration patterns from one province to another. In this article the effects of both internal and international migration flows on long-term population growth in Cuba and in its provinces are discussed. We estimate reproduction and population growth measures using conventional demographic methods, together with the methodology developed by Preston and Wang (2007), based on variable-r methods. Given current demographic patterns in the majority of Cuban provinces, and when migration effects are accounted for, our analysis shows significant negative consequences for the future maintenance of the Cuban population.

Keywords: Cuba. International migration. Domestic migration. Variable-r methods. Stable population models. Population growth. 